\title{
WILEY-VCH
}

DOI: 10.1002/((please add manuscript number))

Article type: Communication

\section{Composite Membrane Formation by Combination of Reaction-Induced and Non- Solvent Induced Phase Separation}

Jamaliah Aburabie, Luis Francisco Villalobos, and Klaus-Viktor Peinemann*

J. H. Aburabie, L. F. Villalobos, Prof. K. V. Peinemann

Advanced Membranes and Porous Materials Center, King Abdullah University of Science and Technology (KAUST), Thuwal 23955-6900, Saudi Arabia

E-mail: klausviktor.peinemann@kaust.edu.sa

Keywords: phase inversion, asymmetric membranes, composite membranes, reaction-induced phase separation

\section{Abstract text}

A novel method of preparing skinned asymmetric membranes with two distinctive layers is described: a top layer composed of chemically cross-linked polymer chains (dense layer), and a bottom layer of non-cross-linked polymer chains (porous substructure). The method consists of two simple steps that are compatible with industrial membrane fabrication facilities. Unlike conventional processes to prepare asymmetric membranes, with this approach it is possible to finely control the structure and functionalities of the final membrane. The thickness of the dense layer can be easily controlled over several orders of magnitude and targeted functional groups can be readily incorporated in it. 


\section{WILEY-VCH}

Main text

The most common fabrication method for preparing membranes is non-solvent induced phase separation (NIPS). The simplicity and scalability of this process makes it the workhorse of the membrane manufacturing industry. ${ }^{[1-4]}$ When dealing with expensive membrane materials or with polymers that are not compatible with NIPS, the preparation of composite membranes is the method of choice. An inexpensive support prepared by NIPS is used, and a thin dense selective layer is applied on top of it by coating or interfacial polymerization. ${ }^{[2,3,5]}$

This Communication describes a new way of composite membrane formation, applicable to cross-linkable polymers. A phase separation induced by a chemical reaction is well known in polymerization processes. However, reaction induced phase separation (RIPS) has never been used in combination with non-solvent induced phase separation for the manufacturing of skinned asymmetric membranes. The new process consists of a combination of reaction- and non-solvent induced phase separation made in two simple subsequent steps. A film of a concentrated polymer solution is immersed in a good solvent for the polymer containing crosslinker molecules. The cross-linking agent present in the solvent induces a surface precipitation, and the fact that a good solvent for the polymer is used ensures that the precipitation is due to the crosslinking reaction. The cross-linking reaction is then stopped by a subsequent immersion in a non-solvent bath without crosslinker. The resulting membrane is a composite-like asymmetric membrane that has a dense top layer consisting of chemically cross-linked polymer chains and a well-defined interface with a porous non-cross-linked support (Figure 1). We described recently a similar process-complexation induced phase separation, or CIPS - that uses the intermolecular complexation of metal ions by polymer chains to form the dense top layer of membranes. ${ }^{[6]}$ In this case the surface precipitation was induced by metal complexation, leading to a top-layer highly loaded with metal ions. RIPS method is not limited to metal complexing polymers, avoids the use of metal ions and 


\section{WILEY-VCH}

facilitates the incorporation of desired organic functionalities in only the top dense layer of membranes.
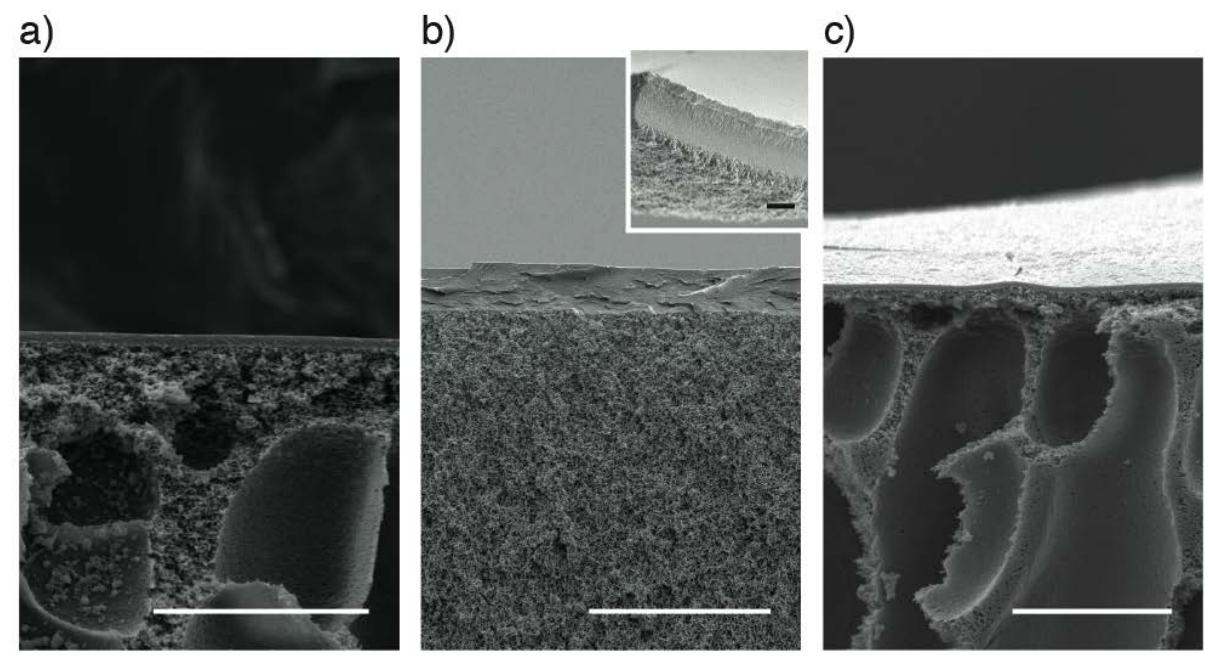

Figure 1. Cross section of a polythiosemicarbazide (PTSC) membranes prepared by RIPS. a) dense layer prepared with 2\% DBX crosslinker for 20 s (top-layer thickness: $370 \mathrm{~nm}$ ). b) dense layer prepared with 10\% DBX crosslinker for 20 s (top-layer thickness: $2000 \mathrm{~nm}$ ). c) dense layer prepared with 5\% Tr-X crosslinker for 20 s (top-layer thickness: $250 \mathrm{~nm}$ ). Scale bars represent $10 \mu \mathrm{m}$ in the main images and $1 \mu \mathrm{m}$ in the inset.

The concept of phase separation, or phase inversion covers a range of different techniques in which a solidification process is initiated and eventually, the polymer precipitates. ${ }^{[2,5]}$ Precipitation can be achieved by shifting the homogenous polymer solution to a nonhomogenous state by processes such as decreasing the solvent concentration through solvent evaporation, ${ }^{[7,8]}$ changing the temperature of the solution, referred as thermally induced phase separation (TIPS), ${ }^{[9]}$ or the introduction of non-solvent to the polymer-solvent system, referred as NIPS. ${ }^{[10]}$ In the case of RIPS, the phase separation is induced by chemical crosslinking of the polymer chains via covalent bonds. A high degree of crosslinking causes the polymer to precipitate out of the solvent (a good solvent for the polymer). A fast crosslinking reaction is essential. It should be faster than the dissolution reaction; otherwise 


\section{WILEY-VCH}

the polymer chains will escape into the bulk of the solution before the crosslinker molecules are able to form a continuous 3-D network of them.

Crosslinking of the polymer chains that form a membrane is a common practice to increase stability or add certain functionality to a membrane. Contrary to the RIPS process, most crosslinking approaches reported so far have been applied as a post treatment to already made polymeric membranes. Once made, membranes are immersed in a crosslinking bath for a specific time and at a certain temperature to ensure a crosslinking reaction. ${ }^{[11,12]}$ Vanherck et al. aimed to reduce the fabrication steps and use of materials by adding the cross-linker directly into the non-solvent bath. ${ }^{[11]}$ This in-situ amine crosslinking was later optimized by Hendrix et al. ${ }^{[13]}$ The crosslinking occurred in the entire membrane because the factor causing the precipitation was the non-solvent and not the crosslinking reaction. In the RIPS process the bath is made of a good solvent for the polymer directing the precipitation to the interface between the polymer solution film and the bath containing the crosslinker molecules. Making the dense layer and porous support in different steps allows more control in the final structure of the membrane and independent optimization of both. The thickness of the dense layer can be easily controlled. Changing the immersion time and the concentration of the cross-linker in the first bath can lead to an increase or decrease in the thickness.

The three general steps to prepare membranes using the RIPS process are: (i) casting of a thin film with the polymer solution; (ii) immersing the polymer film in a first bath of a good solvent for the polymer containing a certain concentration of cross-linker; (iii) placing the resulting film in a non-solvent bath. The second step is the key step, where the polymer chains at the top layer of the casted film are chemically cross-linked creating a thin insoluble solid layer. The formation of this dense layer delays the diffusion of the cross-linker to the rest of the polymer film. The third step is responsible for the formation of a porous support by conventional non-solvent induced phase separation. 


\section{WILEY-VCH}

The most important parameters affecting the formation of the dense layer during the second step are the crosslinker concentration, the immersion time in the first bath, and the choice of cross-linker type. Longer immersion times and higher concentrations produced thicker layers. The thickness of the dense layer could be controlled over several orders of magnitude, from less than $100 \mathrm{~nm}$ to a few micrometers. Moreover, it was possible to add desired functional groups to the active layer of the membrane by choosing the appropriate cross-linker molecules. The size and chemical nature of these molecules had a strong influence on the performance of the final membrane.

The formation of the top layer of asymmetric membranes happens by a phenomenon called gelation. Gelation can be defined as the formation of a three-dimensional network that creates junctions between polymer chains either by chemical crosslinking or physical associations. ${ }^{[2,14,15]}$ Asymmetric membranes which rely on physical associations to form, experience two different types of phase separation: one is gelation induced by H-bonding or crystallization for the skin layer formation and the other is liquid-liquid demixing followed by gelation of the polymer rich phase to form the porous support. Gelation via chemical crosslinking, i.e., covalent binding of polymer chains by means of a chemical reaction, was reported to be unlikely to occur during formation of phase inversion membranes. ${ }^{[15]}$ This communication shows how by careful choice of the experimental parameters it is possible to precipitate the skin layer of a membrane using gelation via chemical cross-linking. RIPS is used for the first time to create covalent bonds to form the dense top layer of asymmetric membranes prepared by phase inversion. Chemical crosslinking gelation offers options to optimize the morphology of the resulting skin layer independently from the porous supportan unlikely feature when physical associations are responsible to form the skin layer.

The RIPS method was developed with the polymer polythiosemicarbazide (PTSC), ${ }^{[16]}$ the structure is shown in (Figure 2a), and the synthesis procedure and characterization are presented in the supporting information. The crosslinkers used are $\alpha, \alpha$ dibromo-p-xylene 


\section{WILEY-VCH}

(DBX) from Sigma-Aldrich and (2,3,6,7,14,15-Hexakis (bromomethyl)-9,10-dihydro-9, 10[1', 2'] benzenoanthracene) ( $\operatorname{Tr}-\mathrm{X})$, which was synthesized in our lab following a procedure reported by Bonaccorsi et al. ${ }^{[17]}$ Details of the $\mathrm{Tr}-\mathrm{X}$ cross-linker synthesis procedure are presented in the supporting information. Structures of both cross-linkers are shown in Figure $2 \mathrm{~b}$ and 2c.

a)<smiles>CNC(=S)NN1CCN(NC(=S)Nc2cccc([CH]c3ccc(C)cc3)c2)CC1</smiles>

b)<smiles>BrCc1ccc(CBr)cc1</smiles>

c)

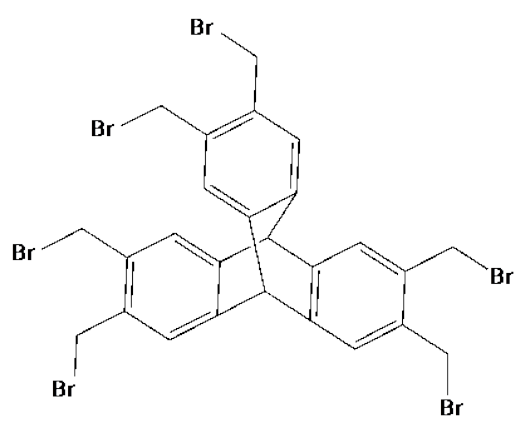

Figure 2. Chemical structure of a) PTSC polymer used for RIPS method, b) DBX crosslinker, c) Tr-X crosslinker.

Recently, PTSC was introduced by our group as a promising material for the preparation of organic solvent nanofiltration membranes. Already prepared NIPS PTSC membranes were post cross-linked with DBX cross-linker. ${ }^{[18]}$ Such membranes showed superior resistance to harsh solvents. Here we report a new approach for preparing asymmetric membranes that uses the same components but the crosslinking is done before the membrane is completely formed to have more tunability of the final product and a more efficient fabrication process. The crosslinking reaction between PTSC and the type of cross-linkers used in this study is a neucleophilic bimolecular $S_{N} 2$ reaction called Menshutkin reaction ${ }^{[19,20]}$ where 


\section{WILEY-VCH}

quarternization of the tertiary amine by alkyl halide takes place. This type of reaction is ideal to test the RIPS process because under the right conditions (i.e. nature of the nucleophile, nature of the leaving group, solvent used, and nature of the substituents bonded to the leaving group ${ }^{[21]}$ ) the reaction kinetics can be very fast-faster than the dissolution kinetics of the polymer in the solvent used. Sufficient amount of cross-links causes phase separation of the three dimensional network of polymer chains. The phase separation happens due to a chemical reaction; consequently the top layer thickness is dependent on variables that affect the reaction rate such as concentration and time. ${ }^{[22]}$

All the membranes presented were made by dissolving PTSC (15 wt\%) in dimethylsulfoxide (DMSO) under heat $\left(65^{\circ} \mathrm{C}\right)$ and stirring for at least 4 hours. Once a homogenous solution was obtained it was left aside to eliminate air bubbles. The polymer solution was then cast on a polyester nonwoven support or a glass plate using a casting knife with $250 \mu \mathrm{m}$ air gap. The polymer film was then immersed in a bath of crosslinker (DBX or Tr-X) dissolved in DMSO for a certain time. Finally, the film was carefully removed from this bath and immersed in a water bath. The residence time in the crosslinking solution had a strong influence on the thickness of the top layer; longer contact time resulted in thicker dense layers. Figure 3 illustrates the change in top layer thickness as a function of immersion time for two crosslinkers (DBX and Tr-X) and also highlights the thickness change with different concentrations of the DBX crosslinker.

As soon as the film gets exposed to the cross-linker bath a dense film forms in less than one second. However, larger immersion times were needed to obtain stable (i.e. thicker) dense layers. The extra time after the appearance of the thin solid layer is the time needed for the cross-linker molecules to diffuse through the newly formed dense barrier and continue to cross-link polymer chains beneath it, growing the layer as a result. Figure $\mathbf{4}$ shows a matrix of SEM images illustrating the effect of immersion time and DBX cross-linker concentration on the dense top layer thickness, which ranges between $100 \mathrm{~nm}$ and $2.5 \mu \mathrm{m}$. The top layer 
thickness can be tuned easily within this range by choosing suitable combination of crosslinker concentration and reaction time.

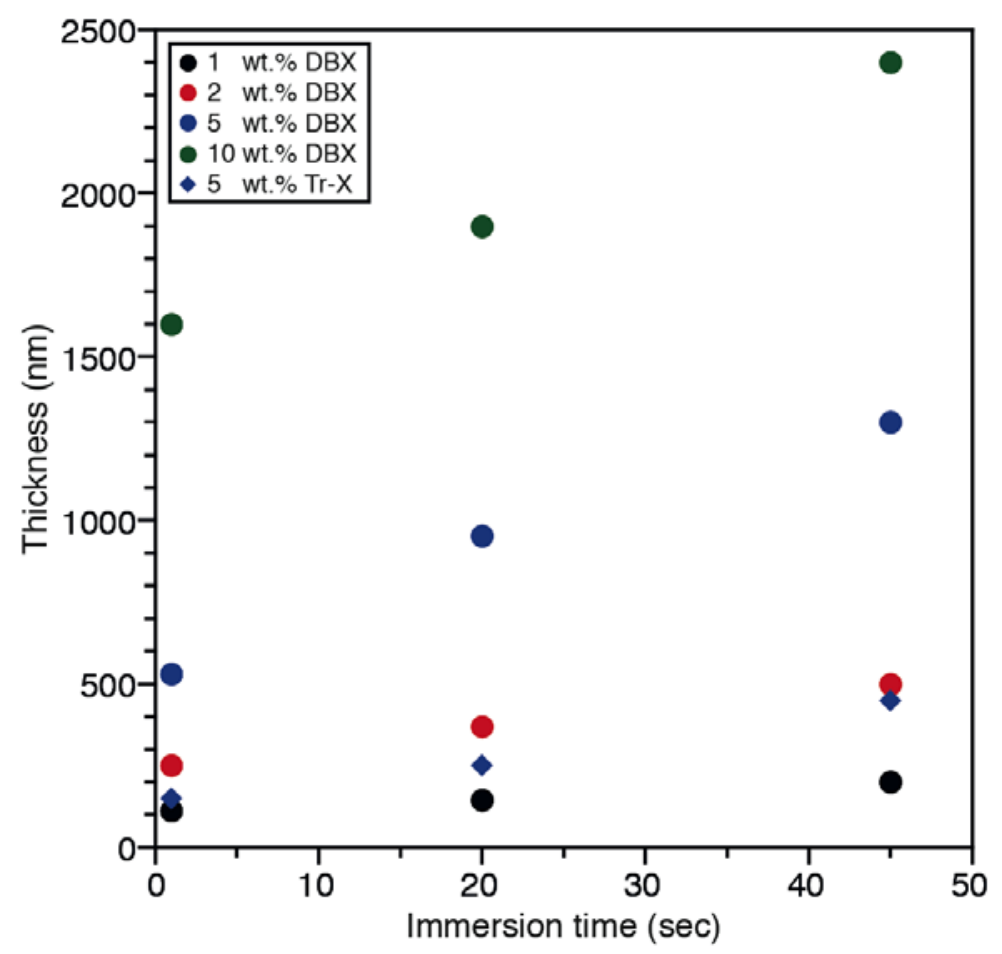

Figure 3. Effect of the crosslinker concentration and immersion time in the dense layer thickness for two different crosslinkers: DBX and Tr-X.

The performance of PTSC membranes prepared with RIPS using different DBX concentrations and immersion times was evaluated by filtering $0.1 \%$ polyethylene glycol (PEG) aqueous solutions in a dead end pressurized stirred filtration cell and measuring the rejection. The results for different molecular weight PEGs are summarized in Figure S12. The membranes showed increased rejection performance depending on the cross-linker concentration and immersion time in the cross-linker bath. For example using $1 \%$ DBX/DMSO as first bath for 20 s showed no rejection for any of the PEGs. With increasing concentration of the cross-linker to $2 \%$ and a short immersion time of one second, the membrane was able to reject only $10 \%$ of the $35 \mathrm{~K}$ PEG. We observed no defects in the dense layer of these two membranes during the SEM analysis, however the low rejections suggest a significant presence of defects in the selective layer. 


\section{WILEY-VCH}

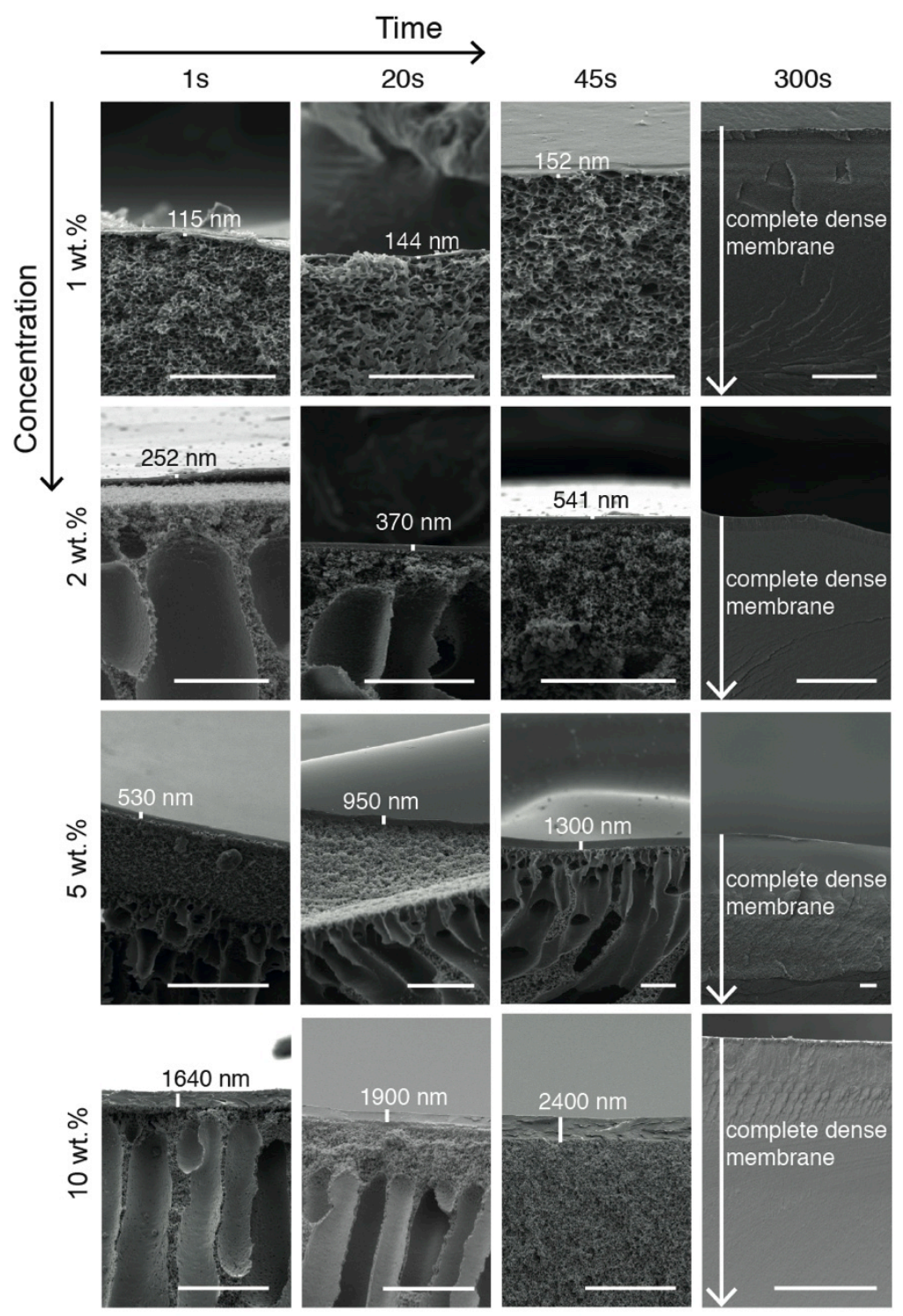

Figure 4. SEM cross section images of membranes prepared by the RIPS method from 15 wt\% PTSC polymer, with different concentrations of DBX crosslinker in DMSO as first bath (values on the left side), the values on the top represent immersion time in crosslinker bath. Scale bars represent $10 \mu \mathrm{m}$.

These defects might be cracks produced during transfer of the delicate support-free thin layers from one bath to the other. Defect-free layers could be obtained for fabrication conditions that produced thicker layers during the first step. Such membranes, prepared with $2 \%$ or $5 \%$ DBX/DMSO and 20 s, showed more than $91 \%$ rejection of the smallest PEG tested (400 Da) and rejected completely (>99\%) rose Bengal (RB, molecular weight $1017 \mathrm{~g} \mathrm{~mol}^{-1}$, 
negative charge) in water. To test their performance in harsh environments we submitted the membrane prepared using 5\% DBX/DMSO and $20 \mathrm{~s}$ to an extra step (immersion in 2\% DBX/acetone for two hours) to crosslink the porous support and make it solvent-stable. The membrane was not able to reject successfully RB in DMSO and DMF, probably due to swelling of the selective layer in these solvents. Nevertheless, successful rejections were obtain for slightly larger molecules: > 99\% rejection of direct red (DR, molecular weight $1373 \mathrm{~g} \mathrm{~mol}^{-1}$, negative charge) in both solvents, and 93\% and 91\% rejections of vitamin B12 (molecular weight $1355 \mathrm{~g} \mathrm{~mol}^{-1}$, neutral charge) in DMSO and DMF respectively, UV-Vis analysis are presented in Figure S13 and S14.

Good rejection results were obtained with membranes prepared using the DBX crosslinker, however the permeances were low. The membranes prepared using 2\% DBX/DMSO (20 s) and 5\% DBX/DMSO (20 s) presented a water permeance of 0.65 , and $0.06 \mathrm{~L} \mathrm{~m}^{-2} \mathrm{~h}^{-1} \mathrm{bar}^{-1}$ respectively (Table 1).

Table1. Permeances for membranes prepared using different parameters

\begin{tabular}{|c|c|c|c|}
\hline Membrane & $\begin{array}{c}\text { Permeance } \\
\left(\mathrm{L} \mathrm{m}^{2} \mathrm{~h}^{-1} \text { bar }^{-1}\right)\end{array}$ & Solvent & Structure \\
\hline NIPS (immersed directly in water bath) & 650 & Water & Porous surface \\
\hline RIPS, 1\% DBX, $20 \mathrm{~s}$ & 191 & Water & Dense top layer with many defects \\
\hline RIPS, $2 \%$ DBX, $1 \mathrm{~s}$ & 10.5 & Water & Dense top layer with few defects \\
\hline RIPS, $2 \%$ DBX, $20 \mathrm{~s}$ & 0.65 & Water & Defect-free top layer \\
\hline RIPS, 5\% DBX, $20 \mathrm{~s}$ & 0.06 & Water & Defect-free top layer \\
\hline RIPS, 5\% Tr-X, $20 \mathrm{~s}$ & 7.7 & Water & Defect-free top layer \\
\hline $\begin{array}{l}\text { RIPS, } 5 \% \text { DBX, } 20 \mathrm{~s}+\text { additional } \\
\text { crosslinking step }\end{array}$ & 0.48 & DMSO & $\begin{array}{c}\text { Defect-free top layer in crosslinked } \\
\text { support }\end{array}$ \\
\hline $\begin{array}{l}\text { RIPS, } 5 \% \text { DBX, } 20 \mathrm{~s}+\text { additional } \\
\text { crosslinking step }\end{array}$ & 0.52 & DMF & $\begin{array}{c}\text { Defect-free top layer in crosslinked } \\
\text { support }\end{array}$ \\
\hline $\begin{array}{l}\text { Isolated layer in alumina support (RIPS, } \\
5 \% \text { DBX, } 20 \mathrm{~s} \text { ) }\end{array}$ & 1.8 & DMSO & Isolated defect-free top layer \\
\hline $\begin{array}{l}\text { Isolated layer in alumina support (RIPS, } \\
5 \% \mathrm{DBX}, 20 \mathrm{~s} \text { ) }\end{array}$ & 4.5 & DMF & Isolated defect-free top layer \\
\hline
\end{tabular}




\section{WILEY-VCH}

It can be assumed that using a bulkier crosslinker would create more interconnected voids (i.e channels) in the matrix of the top selective layer. True to this expectation, a membrane prepared using $5 \%$ of $\mathrm{Tr}-\mathrm{X}$ - a bulky, rigid brominated triptycene ${ }^{[23]}$ —as the crosslinker showed a permeance increase of more than two orders of magnitude in comparison with a membrane made with the same conditions but using DBX-a linear and small crosslinker. The water permeance of the membrane prepared with $\operatorname{Tr}-\mathrm{X}$ was $7.7 \mathrm{~L} \mathrm{~m}^{-2} \mathrm{~h}^{-1} \mathrm{bar}^{-1}$ and the rejection of RB was as high as its DBX counterpart (>99\%). The increase in permeance can be attributed partially to the thinner dense top layer formed when using $\operatorname{Tr}-\mathrm{X}$ (250 nm) compared to the layer obtained with DBX for the same fabrication conditions (950 nm). However, the thickness is not the principal factor. Membranes prepared using DBX and fabrication conditions to obtain a layer of similar thickness (370 nm) still present a permeance more than an order of magnitude lower than the membrane prepared with Tr-X. For any fixed set of fabrication conditions, Tr-X produced thinner layers than DBX (Figure 3). It diffuses slower due to its larger size, resulting in a lower rate of crosslinking.

The top dense layer of membranes prepared via RIPS should be very stable because it is made of cross-linked polymer chains. To test to which extent, a piece of a membrane prepared using a 5\% DBX/DMSO solution and an immersion time of 20 s was submerged in DMSO for 10 days. After some minutes, a transparent layer detached form the nonwoven support and floated on the surface, whereas the non-crosslinked support dissolved completely as for the rest of the time, the layer was maintained. Such thin, transparent layer was stable for the entire duration of the test. Overnight immersion of such membrane in DMSO was repeated and the isolated thin layer was transferred via a glass slide to a water bath for better spreading and carefully placed on top of an inorganic porous alumina support (Anodisc 25, Whatman Ltd, $200 \mathrm{~nm}$ pore size), SEM images of the nanofilm on top of the alumina support are presented in Figure S18. Water was permeated through it using different pressures up to 21 bars; the pressure limit of our set-up (Figure S19). The flux increased linearly with the increase in 


\section{WILEY-VCH}

pressure indicating that the layer did not suffer any compaction or rupture. Further verification of both, the mechanical integrity of the membrane and its stability in harsh solvents was done by permeating solutions of DR in DMSO and DMF and measuring its rejection. More than 99\% rejection was obtained in DMSO and 93\% rejection in DMF. The permeance of the isolated layer was much higher than the same layer on top of its original polymeric support (i.e. before isolation) for both solvents: 3.8 and 8.7 times higher for DMSO and DMF respectively. Digital images of the floating nanofilm, and the nanofilm fixed on the alumina support are provided in Figure S22 and Figure S23, 24 respectively.

In conclusion, a new method, the combination of reaction and non-solvent induced phase separation, was successfully added to the family of phase inversion techniques to prepare composite-like asymmetric membranes. It relies on fast crosslinking reaction rates of polymer chains to form the skin dense layer of the membrane. The method was developed using the PTSC, a polymer which can be cross-linked with a sufficiently high reaction rate to favor the crosslinking reaction and not the dissolution reaction. Future studies will extend this method to other polymers. The two main advantages of this manufacturing method are the tunability of the membrane structure and the possibility to incorporate specific functionalities in the top dense selective layer only. It was demonstrated how a bulky crosslinker can enhance dramatically the performance of the prepared membranes compared to a small one. Besides increasing the interchain spaces inside the dense layer by using a bulky crosslinker, we envision the use of RIPS to incorporate functionalities to help mitigate biofouling, enhance the transport of specific molecules, or capture selectively a compound.

\section{Supporting Information.}

Experimental details; PTSC synthesis and characterization; synthesis of Tr-X crosslinker and characterization; membranes preparation; additional SEM images of membranes made by the RIPS process covering factors such as time of immersion; concentration and type of crosslinker; rejection tests for PEG's and dyes (PDF) and FTIR analysis. 


\section{WILEY-VCH}

\section{Acknowledgements}

King Abdullah University of Science and Technology (KAUST) supported this research. The authors thank Dr. Madhavan Karunakaran for the bromination of Triptycene.

Received: ((will be filled in by the editorial staff))

Revised: ((will be filled in by the editorial staff))

References

Published online: ((will be filled in by the editorial staff))

[1] R. E. Kesting, Synthetic polymeric membranes: a structural perspective. Wiley, New York, 1985.

[2] M. Mulder, Basic Principles of Membrane Technology. 2nd ed.; Kluwer Academic Publishers: Netherlands, 1996.

[3] S. P. Nunes, K.-V. Peinemann (Eds.), Membrane Technology in the Chemical Industry. Wiley-VCH, 2001.

[4] L. K. Wang, J. P. Chen, Y.-T. Hung, N. K. Shammas, Membrane and Desalination Technologies. Springer, 2010.

[5] R. W. Baker, Membrane Technology and Applications. Wiley, 2004.

[6] L. F. Villalobos, M. Karunakaran, K.-V. Peinemann, Nano Lett. 2015, 15, 3166.

[7] H. F. Pierce, J. Biol. Chem. 1927, 75, 795.

[8] J. D. Ferry, Chem. Rev. 1936, 18, 373.

[9] A. J. Castro, US Patents 4,247,498, 1981.

[10] S. Loeb, S. Sourirajan, Sea Water Demineralization by Means of an Osmotic

Membrane. University of California, Department of Engineering, 1960.

[11] K. Vanherck, A. Cano-Odena, G. Koeckelberghs, T. Dedroog, I. Vankelecom, J. Membr. Sci. 2010, 353, 135.

[12] M. F. J. Solomon, Y. Bhole, A. G. Livingston, J. Membr. Sci. 2012, 423, 371.

[13] K. Hendrix，K. Vanherck, I. Vankelecom, J. Membr. Sci. 2012, 421, 15.

[14] J. G. Wijmans, J. Kant, M. Mulder, C. A. Smolders, Polym. 1985, 26, 1539.

[15] S. G. Li, T. Van den Boomgaard, C. A. Smolders, H. Strathmann, Macromolecules 1996, 29, 2053.

[16] T. W. Campbell, E. A. Tomic, J. Polym. Sci. 1962, 62, 379.

[17] P. Bonaccorsi, M. L. Di Gioia, A. Leggio, L. Minuti, T. Papalia, C. Siciliano, A.

Temperini, A. Barattucci, Beilstein J. Org. Chem. 2013, 9, 2410.

[18] J.Aburabie, P. Neelakanda, M. Karunakaran, K.-V. Peinemann, React. Funct. Polym. 2015, 86, 225.

[19] R. W. Taft, Progress in Physical Organic Chemistry. Wiley, 1993.

[20] M. Sola, A. Lledos, M. Duran, J. Bertran, J. L. M. Abboud, J. Am. Chem. Soc. 1991, 113, 2873.

[21] G. Patrick, BIOS Instant Notes in Organic Chemistry. Taylor \& Francis, 2003.

[22] K. A. Connors, Chemical kinetics: the study of reaction rates in solution. John Wiley \& Sons, 1990.

[23] J. H. Chong, M. J. MacLachlan, Chem. Soc. Rev. 2009, 38, 3301. 